\title{
Analyses of Factors Contributing to Vulnerability to Antenatal Periventricular Leukomalacia Induced by Hemorrhagic Hypotension in Chronically Instrumented Fetal Sheep
}

TAKESHI KUSAKA, TADASHI MATSUDA, KAZUHIKO OKUYAMA, KAZUTOSHI CHO, SATORU OKAJIMA, YOSHIYASU KOBAYASHI, AND SEIICHIRO FUJIMOTO

Department of Obstetrics and Gynecology [T.K., T.M., S.O., S.F.], Division of Perinatal Medicine [K.O., K.C.], Hokkaido University Hospital, Hokkaido University School of Medicine, Sapporo, Hokkaido, Japan, and Department of Veterinary Pathology [Y.K.], Obihiro University of Agriculture and Veterinary Medicine, Obihiro, Hokkaido, Japan.

\begin{abstract}
ABST
Our purpose was to determine factors contributing to vulner-
ability to antenatal periventricular leukomalacia (PVL) induced ability to antenatal periventricular leukomalacia (PVL) induced hypotension was induced in 10 fetal sheep by acutely withdrawing $35 \%$ to $40 \%$ of the fetoplacental blood volume at $113 \mathrm{~d}$ gestation. Brains were processed for histologic analysis $6 \mathrm{~d}$ after the insult. Statistical comparisons of physiologic parameters between fetuses suffering from PVL $(n=5)$ and those without PVL $(n=5)$ were performed. Significant correlations were found between induction of PVL and fetal brain weight, changes
\end{abstract}

in fetal mean blood pressure over time, base excess, oxygen content, hematocrit, and plasma arginine vasopressin (AVP) levels in fetal abdominal aortic blood. Brain developmental stage, the magnitude of induced systemic hypotension, and baseline blood oxygen content were important intrinsic factors in the induction of antenatal PVL by hemorrhagic hypotension in premature fetal sheep. (Pediatr Res 51: 20-24, 2002)
Abbreviation
PVL, periventricular leukomalacia

Periventricular leukomalacia (PVL) is necrosis of cerebral white matter displaying a characteristic distribution (i.e. in the white matter dorsal and lateral to the external angles of the lateral ventricles) in the developing human brain (1). The pathogenesis of PVL has not yet been fully clarified, though it has been reported to be closely associated with cerebral palsy as a major causative factor in premature infants $(2,3)$.

We have shown that sudden systemic hypotension is one of the main factors responsible for inducing ischemic injury in periventricular white matter in premature fetuses, as we have succeeded in the induction of antenatal PVL via hemorrhagic hypotension in chronically instrumented fetal sheep (4). Meanwhile, in our previous report, we noticed that some of the fetuses did not have PVL despite receiving the same hemorrhagic insult at the same gestational age. Thus, it was suggested that there must be some intrinsic factors other than hypotension that contribute to the occurrence of antenatal PVL under these

Received March 22, 2001; accepted July 13, 2001.

Correspondence and reprint requests: Dr. Tadashi Matsuda, Department of Obstetrics and Gynecology, Hokkaido University School of Medicine, North 15, West 7, Kita-Ku Sapporo, 060-8638, Japan; e-mail: choku@med.hokudai.ac.jp

Supported by the Ogyaa Donation Foundation in Japan Association of Obstetricians and Gynecologists. experimental conditions. The present study aims to determine factors contributing to vulnerability to antenatal PVL induced by hemorrhagic hypotension in premature fetal sheep.

\section{METHODS}

Animal preparation. With the approval of the Animal Care and Use Committee of Hokkaido University School of Medicine, this study was carried out from January 1998 to April 2000. The preparations and the protocol of this experiment were the same, in large part, as those of our previous study (4). In brief, at $110 \mathrm{~d}$ gestation, a total of 10 Suffolk ewes with timed pregnancies, confirmed by no occurrence of the next sexual excitement after the initial copulation, underwent surgery while anesthetized with intrathecal tetracaine hydrochloride and intravenous ketamine hydrochloride. After laparotomy and hysterotomy, three electrodes were fixed to the fetal chest wall and polyvinyl catheters were placed in the fetal superior vena cava, inferior vena cava, distal abdominal aorta, and amniotic cavity. In case of twin fetuses, only one underwent the experimentation. All catheters and electrodes were exteriorized through a small incision in the flank of each ewe. The ewes were unrestrained and housed in individual cages, with 
free access to water and food throughout the study period. A recovery period of at least $3 \mathrm{~d}$ was allowed before the experiments, and during that time appropriate antibiotics were administered to the mother, fetus, and amniotic cavity.

Measurements of physiologic parameters. Until completion of the study, fetal arterial and amniotic pressure were continuously monitored with a polygraph and recorded on a digital audio tape recorder, a personal computer, or both. All fetal arterial pressure values were corrected for amniotic fluid pressure.

Blood gas data $\left(\mathrm{pH}\right.$, base excess, $\mathrm{P}_{\mathrm{CO}_{2}}, \mathrm{P}_{2}$, and oxygen content), hematocrit values, endocrine hormone [adrenocorticotropic hormone (ACTH), AVP, cortisol, epinephrine, norepinephrine, and dopamine] levels, and osmotic pressure were measured in blood samples $(6.0 \mathrm{~mL})$ taken from the fetal abdominal aorta. Using the heparinized blood samples $(0.5$ $\mathrm{mL}$ ), blood gas data were determined on a blood gas analyzer (Blood Gas System 860, Bayer Diagnostics, Sudbury, UK) and on a hemoximeter. Blood gas data were corrected for maternal rectal temperature. Hematocrit values were measured by centrifugation. The remaining blood samples $(5.5 \mathrm{~mL})$ were supplemented with ethylenediaminetetraacetic acid-2 potassium (EDTA-2 K) for hormonal assay and osmotic pressure measurement, were centrifuged immediately after collection, and the separated plasma samples were stored at $-80^{\circ} \mathrm{C}$ until assayed. ACTH, AVP, and cortisol levels were determined, using commercially available kits by IRMA (ACTH-II IRMA kit, Mitsubishi Petrochemical, Tokyo, Japan), RIA (AVP-RIA kit, Mitsubishi Petrochemical, Tokyo, Japan), and fluorescence polarization immunoassay (Gamma Coat Cortisol kit, Clinical Assay, Cambridge, Massachusetts), respectively (5-7). Epinephrine, norepinephrine, and dopamine levels in fetal plasma were determined by high-pressure liquid chromatography (HPLC; Autoanalyzer, HLC-725CA, Tosoh Inc, Tokyo, Japan) (8). Osmotic pressures were determined by a freezing point depression method (Autoanalyzer, OM-6030, Arkray, Inc, Tokyo, Japan).

Estimation of fetoplacental blood volume. At $>48 \mathrm{~h}$ after the operation, an isovolemic exchange transfusion was performed on each fetus in which 30 to $40 \mathrm{~mL}$ (v) of blood was removed over 15 to $20 \mathrm{~min}$ at a constant rate from the inferior vena cava catheter while simultaneously replacing the volume through the abdominal aorta catheter with heparinized fresh plasma from another fetal sheep. Hematocrit values were measured before and after exchange transfusion. Fetoplacental blood volume $(\mathrm{V})$ was calculated according to the following formula: $\mathrm{V}=\mathrm{v} / \mathrm{ln}$ (Hematocrit value before transfusion/ Hematocrit value after transfusion) (9). After the exchange transfusion, the removed and heparinized fetal blood was centrifuged at $1,000 \times g$ for $10 \mathrm{~min}$, and the separated erythrocytes were suspended in sodium chloride solution adjusted to the same volume as the exchange volume and returned to the fetuses over $5 \mathrm{~h}$.

Experimental protocol. At 113 days' gestation, the following experiments were conducted in all 10 fetuses. To induce systemic hypotension in the fetuses, $35 \%$ to $40 \%$ of the fetoplacental blood volume was withdrawn over $20 \mathrm{~min}$ at a constant rate from the inferior vena cava catheter, and at $24 \mathrm{~h}$ after bleeding, the blood was returned to the fetuses over $5 \mathrm{~h}$.

The fetal mean blood pressure and heart rate values at each time point represent averages obtained every $5 \mathrm{~min}$. To obtain blood gas data, hematocrit values, endocrine hormone levels, and osmotic pressures, abdominal aortic blood was sampled just before bleeding and at 20 and $60 \mathrm{~min}, 2,4,6,12,18$, and $24 \mathrm{~h}$ after bleeding, and at completion of the return the removed blood. After each sampling, an equivalent volume of maternal heparinized blood was infused into the fetus through the venous catheter.

Neuropathologic examination. Six days after the initial insult, cesarean sections were performed. Under anesthesia, fetal brains were perfused with $10 \%$ neutralized buffered formalin for fixation, removed, and weighed. The cerebral hemispheres were cut into 4 standardized coronal sections at the level of the frontal lobe, the anterior basal ganglia, the mamillary bodies, and the occipital lobe. Multiple additional sections of the cerebellum, midbrain, pons, medulla oblongata, and spinal cord were also made. After macroscopic observation of each section, histopathologic evaluation was performed on 4- $\mu \mathrm{m}$ sections stained with hematoxylin and eosin.

On the basis of the criteria of Banker and Larroche, PVL was defined as the presence of scattered round neuroaxonal swelling and/or focal coagulation necrosis with infiltration by microglia/macrophages, localized within the deep white matter around the lateral ventricles $(1,3)$. As a result of the neuropathologic diagnosis, the fetuses were divided into two groups, a PVL group $(n=5)$ and a non-PVL group $(n=5)$, and the changes over time in physiologic parameters were statistically compared between the two groups.

Statistical analysis. All values are expressed as means \pm SD. Repeated-measures ANOVA was performed for statistical comparison of changes over time in physiologic parameters in the two groups, and if significant changes were suggested over time, Dunnett's test was performed to test for significant changes from the values just before hemorrhagic insults. Differences in continuous values between the two groups were assessed using the Wilcoxon signed-rank test with two-tailed $p$ values. A $p$ value $<0.05$ was considered significant.

\section{RESULTS}

On continuous monitoring of the parameters, all 10 fetuses were in good condition; there were no occasions when the mean arterial pressure was $<35 \mathrm{mmHg}$ or heart rate was $<100$ beats per minute over $3 \mathrm{~min}$ during the study period, except immediately after the insults. Fetal $\mathrm{pH}$ was never $<7.25$ and $\mathrm{PO}_{2}$ was never $<10 \mathrm{mmHg}$. Neither intrauterine infection nor threatened premature labor occurred. All fetuses were available for physiologic and neuropathologic assessment.

Neuropathologic examination of the central nervous system (CNS) demonstrated that five of the 10 fetuses had PVL, characterized predominantly by axonal swelling and/or focal necrosis with activated microglia/macrophages within the deep white matter around the lateral ventricles. Except for PVL lesions, there were no histopathologic changes within the CNS in any of the 10 fetuses examined. 
Table 1. Comparison of basic fetal characters between the PVL and the non-PVL groups

\begin{tabular}{lcc}
\hline & $\begin{array}{c}\text { PVL group } \\
(n=5)\end{array}$ & $\begin{array}{c}\text { Non-PVL group } \\
(n=5)\end{array}$ \\
\hline Singleton/twin pregnancy & $2 / 3$ & $4 / 1$ \\
Gender (male/female) & $3 / 2$ & $3 / 2$ \\
Fetoplacental blood volume (mL) & $225 \pm 31$ & $258 \pm 73$ \\
Withdrawn blood volume (\%) & $37.5 \pm 2.3$ & $37.8 \pm 1.0$ \\
Brain weight (g) & $39.6 \pm 2.6$ & $45.5 \pm 3.5^{*}$ \\
Body weight (kg) & $2.60 \pm 0.14$ & $3.03 \pm 0.34$ \\
Brain/body weight ratio (\%) & $1.50 \pm 0.11$ & $1.52 \pm 0.20$ \\
\hline
\end{tabular}

Categoric variables are shown as number of positive cases and continuous variables are expressed as means $\pm \mathrm{SD}$. The values of body and brain weight were measured at 6 days after the hemorrhagic insult.

$* p<0.05$.

Table 1 shows the results of the statistical analysis of basic fetal characteristics in the two groups. The brain weights in the PVL group were significantly lower than those in the non-PVL group, whereas there was no significant difference between the two groups in the brain/body weight ratio. No significant differences between the two groups were found in fetal number, sex, estimated fetoplacental blood volume, and actual blood volume withdrawn. Although the difference was not statistically significant, the percentage of twin pregnancy in the PVL group tended to be higher than those in the non-PVL group.

The comparisons between the two groups in the prehemorrhage values of physiologic factors are summarized in Table 2. Hematocrit and oxygen content values in the PVL group were significantly lower than those in the non-PVL group at the time of pre-hemorrhagic insult, whereas there were no differences between the two groups in values of heart rate, $\mathrm{pH}, \mathrm{PCO}_{2}$, and $\mathrm{Po}_{2}$.

Figures 1, 2, and 3 display the changes in physiologic parameters in the two groups over the course of the experiment, limited only to some informative parameters. Significant differences in changes in parameters over the time between the two groups were found in mean blood pressure $(p<0.01)$, base excess $(p<0.05)$, oxygen content $(p<0.01)$, hematocrit values $(p<0.05)$, and plasma AVP levels $(p<0.01)$, but not in other parameters including heart rate, $\mathrm{pH}, \mathrm{PCO}_{2}, \mathrm{Po}_{2}$, plasma osmotic pressure, ACTH, cortisol, epinephrine, norepinephrine, and dopamine levels during the experimental period.

Table 2. Comparison of pre-hemorrhage values in physiologic factors between the PVL and the non-PVL groups

\begin{tabular}{lcc}
\multicolumn{3}{c}{ factors between the PVL and the non-PVL groups } \\
& $\begin{array}{c}\mathrm{PVL} \text { group } \\
(n=5)\end{array}$ & $\begin{array}{c}\text { Non-PVL group } \\
(n=5)\end{array}$ \\
\hline Heart rate (beats/min) & $198 \pm 15$ & $188 \pm 13$ \\
Mean blood pressure $(\mathrm{mm} \mathrm{Hg})$ & $41.2 \pm 2.3$ & $41.3 \pm 2.5$ \\
$\mathrm{pH}$ & $7.40 \pm 0.03$ & $7.41 \pm 0.01$ \\
$\mathrm{Base}$ excess & $6.6 \pm 1.6$ & $6.6 \pm 3.7$ \\
$\mathrm{PCO}_{2}(\mathrm{~mm} \mathrm{Hg})$ & $52.7 \pm 5.1$ & $50.1 \pm 5.3$ \\
$\mathrm{Po}_{2}(\mathrm{~mm} \mathrm{Hg})$ & $18.5 \pm 3.6$ & $21.6 \pm 3.2$ \\
$\mathrm{Hematocrit}(\%)$ & $29.1 \pm 1.6$ & $33.1 \pm 2.8^{*}$ \\
Oxygen content $(\mathrm{m} \mathrm{mol} / \mathrm{L})$ & $2.7 \pm 1.0$ & $4.2 \pm 0.5^{*}$ \\
\hline
\end{tabular}

Continuous variables are expressed as means $\pm \mathrm{SD}$.

$* p<0.05$.
When blood withdrawal was started, the mean blood pressure abruptly decreased and then rapidly recovered within 40 min in both groups (Fig. 1B). Comparing changes in mean blood pressure during hemorrhage between the two groups, no significant differences were found in the lowest values of mean blood pressure, but the magnitude $(p<0.05)$ and the rate $(p<$ 0.05 ) of blood pressure decrease versus the values just before the hemorrhagic insult were higher in the PVL than in the non-PVL group (Table 3).

Base excess tended to decrease from just after hemorrhage to $6 \mathrm{~h}$ later $(6.6 \pm 1.5$ to $3.9 \pm 3.0)$ in the PVL group, whereas in the non-PVL group a slight increase was seen during the same period (Fig. 2A). Oxygen content decreased gradually from the time just after hemorrhage to the reinfusion of removed blood in both groups. During the experimental course, oxygen contents were significantly lower in the PVL group than in the non-PVL group (Fig. 2B). Hematocrit changes were similar to those in oxygen content in both groups (Fig. 2C).

Plasma AVP levels were apparently higher in the PVL group than in the non-PVL group, reaching the highest value at 20 min after hemorrhage in both groups (Fig. 3). The peak values were $33.4 \pm 20.6 \mathrm{pg} / \mathrm{mL}$ in the PVL group and $12.5 \pm 5.7$ $\mathrm{pg} / \mathrm{mL}$ in the non-PVL group and were significantly elevated in the PVL group compared with the non-PVL group $(p<0.01)$.
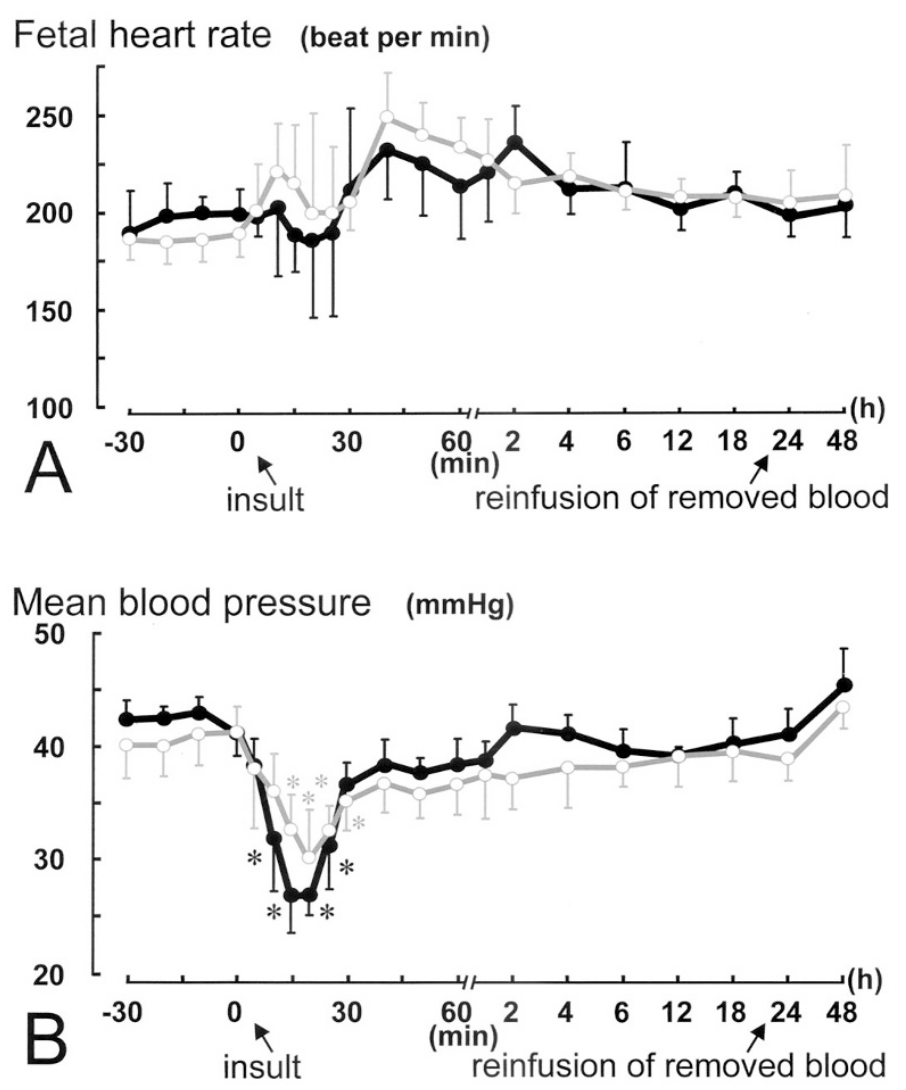

Figure 1. $A$, Comparison of changes over time in fetal heart rate between PVL group and non-PVL group. $B$, Comparison of changes over time in mean blood pressure between the two groups $(p<0.01$, repeated-measures ANOVA). There was a significant difference in the mean blood pressure drop between the two groups (Table 1). $\bullet$, PVL group; ○, non-PVL group. ${ }^{*} p<$ 0.05 (Dunnett's test) compared with the preinsult value. 
Table 3. Comparison of mean blood pressure during acute hemorrhagic hypotension between the PVL and the non-PVL groups

\begin{tabular}{lcc}
\hline \multicolumn{1}{c}{ Mean blood pressure } & $\begin{array}{c}\text { PVL group } \\
(n=5)\end{array}$ & $\begin{array}{c}\text { Non-PVL group } \\
(n=5)\end{array}$ \\
\hline Pre-hemorrhage values (mm Hg) & $41.2 \pm 2.3$ & $41.3 \pm 2.5$ \\
Lowest intra-hemorrhage values (mm Hg) & $25.2 \pm 1.6$ & $29.8 \pm 4.2$ \\
Differences between pre- and lowest & $16.0 \pm 2.1$ & $11.5 \pm 2.9^{* *}$ \\
$\quad$ intra-hemorrhage values (mm Hg) & & \\
Rate of maximal decrease versus & $38.8 \pm 3.9$ & $28.0 \pm 7.6^{*}$ \\
$\quad$ Pre-hemorrhage values (\%) * & & \\
\hline All data are expressed as means \pm SD. \\
$*$ Values (\%) were calculated as follows: (Pre-hemorrhage value - Lowest \\
intra-hemorrhage values) $100 /$ Pre-hemorrhage value. \\
$\quad * * p<0.05$.
\end{tabular}

\section{DISCUSSION}

In this study, significant differences in parameters between the PVL and the non-PVL groups were found in fetal brain weight, pre-hemorrhage values of oxygen content and hematocrit, changes in fetal mean blood pressure over time, as well as base excess, oxygen content, hematocrit, and plasma AVP levels in fetal abdominal aortic blood.

Fetal brain weight $6 \mathrm{~d}$ after the insult was significantly lower in the PVL group than in the non-PVL group. This difference was not considered to be the consequence of PVL because PVL generally does not impair brain growth within a few days after induction $(1,3)$ and there were no difference between the two groups in the brain/body weight ratio (Table 1). It has been reported that the increase of brain weight in fetal sheep spurts around 95 to $110 \mathrm{~d}$ gestation and is significantly associated with neuroglial multiplying and myelinating activity in cerebral white matter $(10,11)$, suggesting that the individual differences in fetal brain weight at $113 \mathrm{~d}$ gestation (the day of insult) should be high. Moreover, in this time window, the fetal cerebral white matter would be susceptible to ischemic insults because rapidly differentiating/myelinating oligodendroglia are vulnerable to environmental changes, such as deprivation of oxygen or glucose supply $(3,10)$. Therefore, it is possible that the brains in the PVL group were in the midst of myelinating burst and under the critical stage of differentiation/myelination to hemorrhagic hypotension, though not proven by the routine histopathology.

Oxygen content and hematocrit values in the PVL group were significantly lower than those in the non-PVL group over the experimental period (Fig. 2, $B$ and $C$ ). This difference in oxygen content was thought to mainly depend on initial hematocrit values (Table 2) because oxygen content changed in parallel with hematocrit values in both groups and no significant difference was found in $\mathrm{PaO}_{2}$ over time. Hematocrit values reportedly increase with developmental age in fetuses $(12,13)$. Thus, hematocrit differences between the two groups may be attributed to the fetal maturity, as observed in fetal brain weight. Hypoxia observed in the PVL group was not so severe as to cause acidemia, though base excess decreased for $6 \mathrm{~h}$ after the insult (Fig. 2A). Even if not particularly severe, differences in oxygen content in fetal blood might be related to the genesis of ischemic brain injury under the condition of
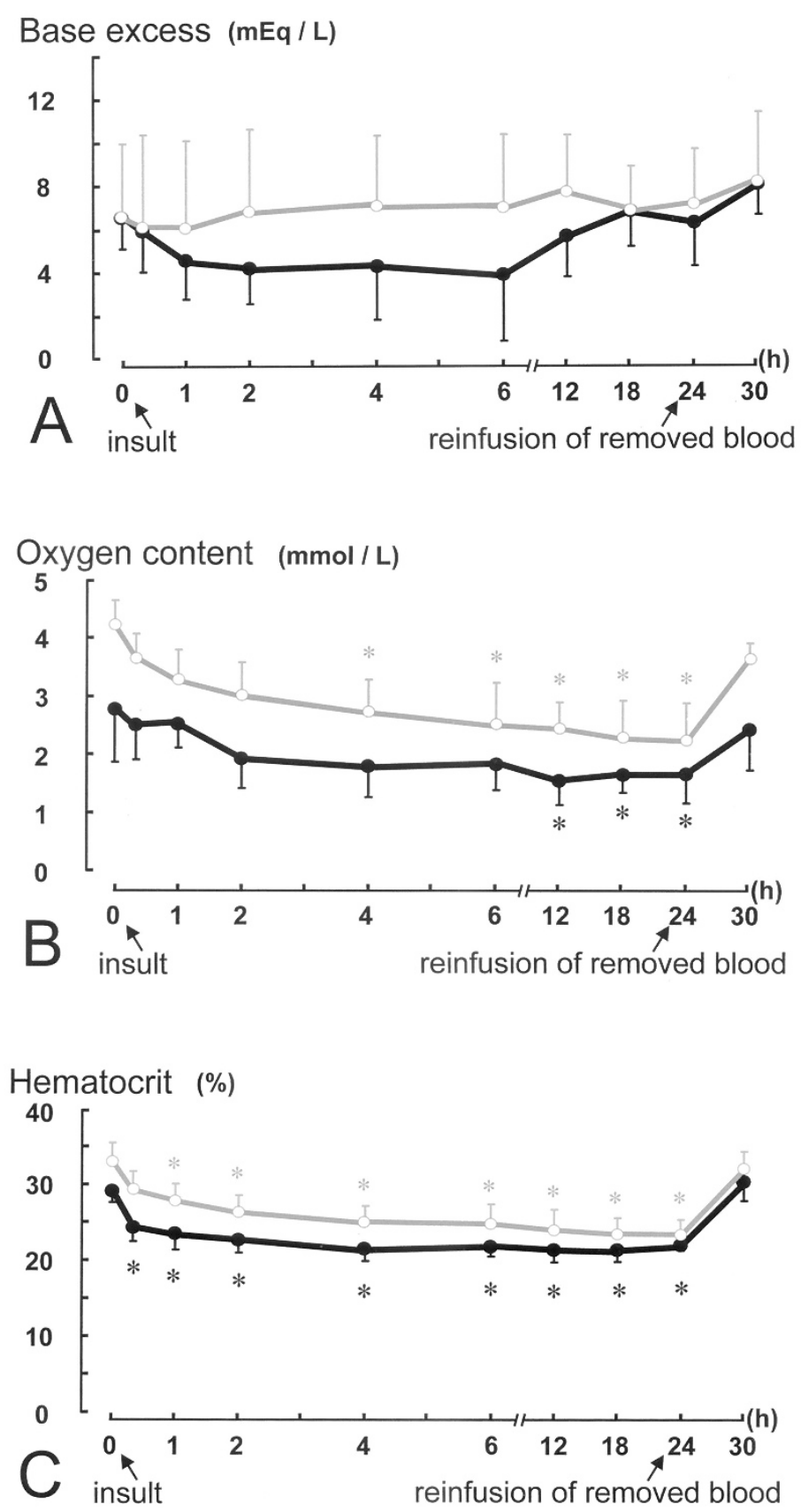

Figure 2. Comparison of changes over time in base excess, oxygen content, and hematocrit values of fetal abdominal aortic blood between the PVL group and the non-PVL group. $A$, base excess $(p<0.05) ; B$, oxygen content $(p<$ $0.01) ; C$, hematocrit $(p<0.05) . p$ values in parentheses show results of repeated-measures ANOVA respectively. $\bullet$, PVL group; $\bigcirc$, non-PVL group. $* p<0.05$ (Dunnett's test) compared with the preinsult value.

decreased fetal cerebral blood flow caused by systemic hypotension. However, as we reported previously (4), the decrease of oxygen content produced by severe anemic change by isovolemic exchange transfusion alone did not induce histopathologic findings, including PVL, in premature fetal brain. Therefore, the decrease of oxygen content was considered to be an exacerbating factor rather than a primary factor in the induction of antenatal PVL by hemorrhagic hypotension in fetal sheep. 


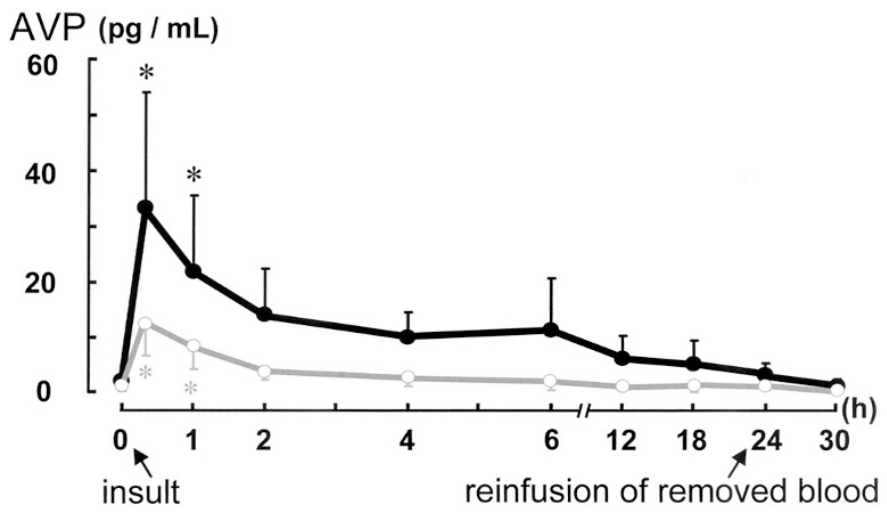

Figure 3. Comparison of changes over time in AVP levels of fetal abdominal aortic blood between the PVL group and the non-PVL group. Plasma AVP levels were apparently higher in the PVL group than in the non-PVL group ( $p$ $<0.05$, repeated-measures ANOVA). The peak plasma AVP values in the PVL group were significantly higher than those in the non-PVL group ( $p<$ 0.05 ). - PVL group; $\odot$, non-PVL group. ${ }^{*} p<0.05$ (Dunnett's test) compared with the preinsult value.

The magnitude and the rate of blood pressure decrease versus the values just before the hemorrhagic insult were higher in the PVL group than in the non-PVL group (Fig. $1 B$ and Table 3). Papile et al. (14) demonstrated that decreases in cerebral blood flow are linearly correlated with mean blood pressure below the lower limit of autoregulation in fetal sheep, supporting the importance of the degree of systemic hypotension in the occurrence of antenatal PVL. In acute hemorrhagic insults in fetal sheep, baroreceptor regulation and secretion of vasoactive hormones will operate to increase peripheral vasoconstriction to maintain arterial mean blood pressure $(15,16)$. However, there was no significant difference in actual blood volume withdrawn (Table 1) and changes with time of heart rate (Fig. $1 \mathrm{~A}$ ) and plasma catecholamine levels between the two groups. This result may be mainly because differences in the responses to hemorrhage in plasma epinephrine and norepinephrine levels in each group were too small to detect compared with those in other reports using more mature fetuses at $119 \sim 135 \mathrm{~d}$ gestation $(15,17)$. The defensive mechanisms to acute hemorrhage are considered to be altered with fetal prematurity (16). It is necessary to analyze the relationship between fetal development and these compensatory reactions to hemorrhagic hypotension.

Plasma AVP levels, especially at their peak, were significantly elevated in the PVL group compared with the non-PVL group (Fig. 3). However, no significant difference was found in osmotic pressure changes between the two groups. Thus, it is likely that the difference in plasma AVP levels between the two groups reflected the degree of systemic hypotension induced by hemorrhage. It has been reported that the plasma AVP elevation correlates exponentially with a decreased blood pressure ratio (18). Accordingly, plasma AVP levels should be recognized as a dependent factor influencing the degree of hemorrhagic hypotension.

Twin pregnancy has been reported to be one of the major clinical factors associated with antenatal PVL (2). In this study, however, the association could not be documented, though the percentage of twin pregnancy in the PVL group was three times as high as that in the non-PVL group. This ratio was possibly owing to the paucity of the entry number of experimental animals. Because the body weights of sheep fetuses in twins tended to be smaller than those in singletons, twinning might effect fetal brain growth and development. However, we could not find any significant differences between twin $(n=4)$ and singleton fetuses $(n=6)$ in fetal brain weight, the magnitude of systemic hypotension, and baseline blood oxygen content in this experiment (data not shown).

Based on these considerations, intrinsic factors producing vulnerability to antenatal PVL induction by hemorrhagic hypotension in premature fetal sheep are the stage of brain development, the magnitude of systemic hypotension, and baseline blood oxygen content. Changes in base excess, hematocrit, and plasma AVP levels would be also important as constituent or dependent factors influencing the three major factors. Brain developmental stage could be an essential factor in producing vulnerability, whereas systemic hypotension is a requisite trigger and hypoxemia an exacerbating factor in the genesis of antenatal PVL.

Acknowledgments. The authors thank Kunihiko Kobayashi, MD, Department of Pediatrics, Hokkaido University School of Medicine, for his review of and suggestions regarding the manuscript. We also thank Tatsuro Kishida, MD, Department of Obstetrics and Gynecology, Hokkaido University School of Medicine, and Yoshinori Matsumoto, MD, Hokkaido Social Work Association Obihiro Hospital, for their technical assistance.

\section{REFERENCES}

1. Banker BQ, Larroche JC 1962 Periventricular leukomalacia of infancy: a form of neonatal axonic encephalopathy. Arch Neurol 7:386-410

2. Kuban KCK, Leviton A 1994 Cerebral palsy. N Engl J Med 330:188-195

3. Volpe JJ 1995 Hypoxic-ischemic encephalopathy: neuropathology and pathogenesis, periventricular leukomalacia. In: Volpe JJ (ed) Neurology of the Newborn,. 3rd ed. WB Saunders, Philadelphia, pp 291-299

4. Matsuda T, Okuyama K, Cho K, Hoshi N, Matsumoto Y, Kobayashi Y, Fujimoto S 1999 Induction of antenatal periventricular leukomalacia by hemorrhagic hypotension in the chronically instrumented fetal sheep. Am J Obstet Gynecol 181:725-730

5. Alexander DP, Britton HG, Forsling ML, Nixon DA, Ratcliffe JG 1974 Pituitary and plasma concentrations of adrenocorticotropin, growth hormone, vasopressin and oxytocin in fetal and maternal sheep during the latter half of gestation and the response to haemorrhage. Biol Neonate 24:206-219

6. Drummond WH, Rudolph AM, Keil LC, Gluckman PD, MacDonald AA, Heymann MA 1980 Arginine vasopressin and prolactin after hemorrhage in the fetal lamb. Am J Physiol 238:E214-E219

7. Ayers GJ, Clark J, Burnett D 1989 An evaluation of the Abott $\mathrm{TD}_{\mathrm{x}}$ serum cortisol assay. Ann Clin Biochem 26:294-295

8. Jones CM, Rose JC, Kelly RT, Hargrave BY 1985 Catecholamine responses in fetal lambs subjected to hemorrhage. Am J Obstet Gynecol 151:475-458

9. Brace RA 1983 Blood volume and its measurement in the chronically catheterized sheep fetus. Am J Physiol 244:H487-H494

10. McIntosh GH, Gaghurst KI, Potter BJ, Hetzel BS 1979 Foetal brain development in the sheep. Neuropathol Appl Neurobiol 5:103-114

11. Barlow RM 1969 The foetal sheep: morphogenesis of the nervous system and histochemical aspects of myelination. J Comp Neurol 135:249-261

12. Shields LE, Widness JA, Brace RA 1993 Restoration of fetal red blood cells and plasma proteins after a moderately severe hemorrhage in the ovine fetus. Am J Obstet Gynecol 169:1472-1478

13. Bratteby LE 1968 Studies on the erythro-kinetics in infancy: red cell volume of newborn infants in relation to gestational age. Acta Paediatr Scand 57:132-136

14. Papile LA, Rudolph AM, Heymann MA 1985 Autoregulation of cerebral blood flow in the preterm fetal lamb. Pediatr Res 19:159-161

15. Meyers RL, Paulick RP, Rudolph CD, Rudolph AM 1991 Cardiovascular response to acute, severe hemorrhage in fetal sheep. J Develop Physiol 15:189-197

16. Morin III FC, Weiss KI 1992 Fetal circulation: response of the fetal circulation to stress. In: Polin RA, Fox WW (eds) Fetal and Neonatal Physiology,. 1st ed. WB Saunders, Philadelphia, pp 620-629

17. Jones CM, Rose JC, Kelly RT, Hargrave BY 1985 Catecholamine responses in fetal lambs subjected to hemorrhage. Am J Obstet Gynecol 475-478

18. Baylis PH 1987 Osmoregulation and control of vasopressin secretion in healthy humans. Am J Physiol 253:R671-R678 